\title{
CANCER
}

\section{Special delivery service}

\begin{abstract}
Glioblastoma multiforme (GBM) is the most common and the most aggressive form of brain tumour. The infiltrative nature and cellular heterogeneity of GBM make this cancer particularly hard to treat as many therapies fail to penetrate brain tissue deep enough to reach the tumour cells. Indeed, the median survival when surgery, radiotherapy and chemotherapy are combined is just over 1 year.

Now, in a study in PNAS, Saltzman and colleagues describe a promising strategy for the treatment of GBM involving specially designed poly(lactide-co-glycolide) (PLGA) nanocarriers loaded with dithiazanine iodide (DI), a US Food and Drug Administration (FDA)approved antihelminthic, that targets brain cancer stem cells (BCSCs). The researchers were able to deliver
\end{abstract}

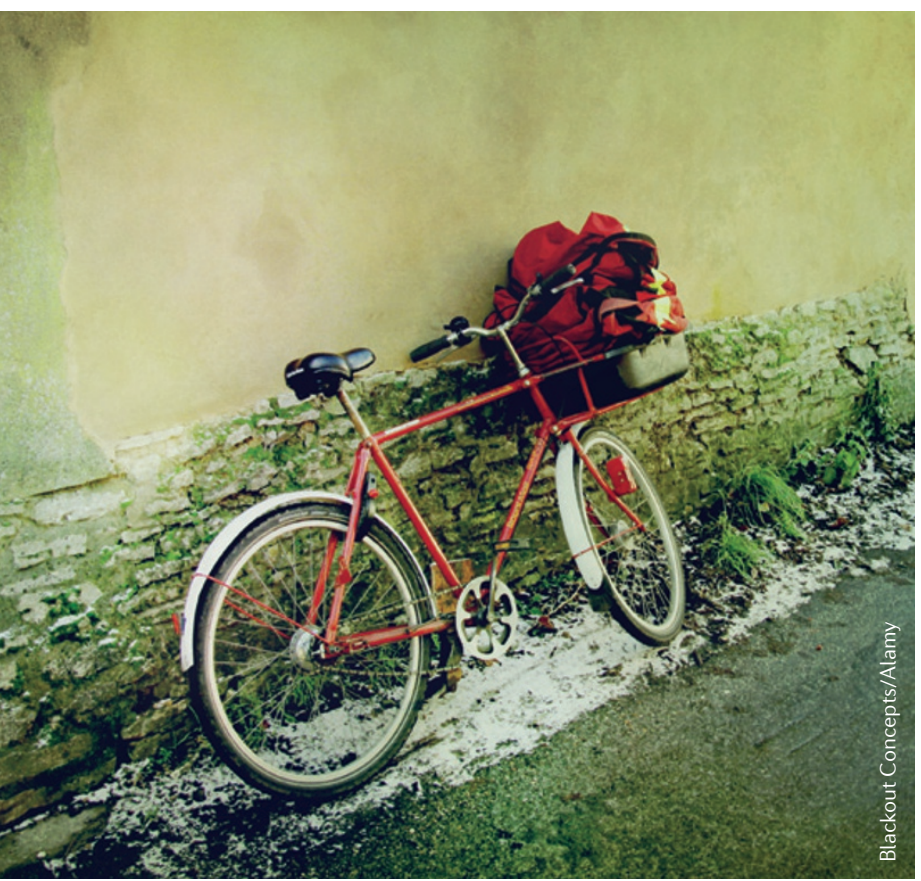

these nanocarriers deep into animal brain tissue by using an optimized convection-enhanced delivery (CED) protocol, and found that they significantly increased the survival of rats bearing BCSC-derived xenografts.

CED is a direct method for drug delivery into the brain through intraparenchymal microcatheters, and previous studies have shown that the depth of penetration of locally delivered therapeutic agents can be improved by CED. The drugs are infused into the brain under a positive pressure gradient that creates bulk fluid movement in the brain interstitium. However, the delivery of drugs in solution by means of CED has not resulted in therapeutic benefits as the short half-lives of the drugs in the brain means that the effects disappear when infusion stops.

Loading drugs into polymeric nanoparticles that protect them from clearance and allow long-term release of active agents could overcome this issue. However, most nanoparticles are not small enough to move through the brain's interstitial spaces. The authors of this study designed PLGA nanoparticles that are smaller ( $\sim 70 \mathrm{~nm}$ diameter) and less prone to aggregation than standard $(\sim 140 \mathrm{~nm}$ diameter) PLGA nanoparticles by using ethyl acetate as a solvent and trehalose as an excipient.

By radiolabelling the two types of nanoparticles the authors were able to track them during the CED procedure using noninvasive positron emission tomography imaging. They found that the volume of distribution of the smaller nanoparticles, in either rat or pig brain, was improved up to sevenfold compared with standard nanoparticles. Moreover, when the smaller-sized nanoparticles were loaded with the chemotherapeutic agent paclitaxel, the survival of rats bearing intracranial tumours was longer than for rats receiving free paclitaxel or standard-sized paclitaxel-loaded nanoparticles.

To further assess the therapeutic potential of these brain-penetrating nanoparticles the authors screened 2,000 FDA-approved compounds for ones that potently inhibited the proliferation and self-renewal of BCSCs in cell culture. Of the 32 hit compounds identified, they loaded the antihelminthic cyanine dye DI onto their brain-penetrating nanoparticles.

When these were delivered by CED into rats inoculated with GS5 a well-characterized BCSC line that leads to the development of tumours that closely resemble human GBM - a significant increase in median survival time was observed after a single treatment. Interestingly, when two other compounds that exhibited similar half-maximal inhibitory concentration $\left(\mathrm{IC}_{50}\right.$ ) values against BCSCs to DI were loaded and delivered: the cardiac glycoside digoxin and the antibiotic anisomycin, no survival benefits were observed.

Thus, although the in vivo efficacy of the compounds that inhibit BCSC proliferation and self-renewal is unpredictable from the cell culture assays, the results with DI suggest that these brain-penetrating nanoparticles represent a promising drug delivery platform that could be applicable to other central nervous system diseases.

Monica Hoyos Flight

ORIGINAL RESEARCH PAPER Zhou, J. et al. Highly penetrative, drug-loaded nanocarriers improve treatment of glioblastoma. Proc. Natl Acad. Sci. USA 110, 11751-11756 (2013) 